\title{
Evolutionary pressures in emerging societies of secondary users in cognitive radio networks
}

\author{
Anna Wisniewska \\ Computer Science Department \\ City University of New York, GC \\ New York, NY 10016, U.S.A \\ awisniewska@gradcenter.cuny.edu
}

\author{
Bilal Khan \\ Department of Sociology \\ University of Nebraska-Lincoln \\ Lincoln, NE 68583, U.S.A \\ bkhan2@unl.edu
}

\author{
Ala Al-Fuqaha \\ Computer Science Department \\ Western Michigan University \\ Kalamazoo, MI 49008, U.S.A \\ ala.al-fuqaha@wmich.edu
}

\author{
Kirk Dombrowski \\ Department of Sociology \\ University of Nebraska-Lincoln \\ Lincoln, NE 68583, U.S.A \\ kdombrowski2@unl.edu
}

\begin{abstract}
Wireless communication is an increasingly ubiquitous and important aspect of the digital ecosystem. In the face of rapid growth in the population of Internet of Things reached 4+ billion devices in 2014, and is expected to continue to grow, reaching 25 billion by 2020 , the limited capacity of radio spectrum is likely to reach saturation. In this paper, we show that evolutionary pressures in $\mathrm{CR}$ societies necessarily drive the emergence of more advanced sensing capabilities, and correspondingly more sophisticated models of resource sharing. We put forth four evolutionary stages for CR societies, based on well-established biological analogues, and demonstrate that at each stage of $\mathrm{CR}$ evolution, a subpopulation that is able to engage more advanced sensing capabilities and couse strategies is able to better extract greater utility from spectrum resources. In this manner, we see that each stage of CR evolution prepares the way for the next: the present societies of nonforagers facilitate the emergence of foragers; foragers give way to contention-sensing rational CR societies; these, in turn, will likely facilitate the emergence of sociality. Each evolutionary stage is enabled by advances in sensory capabilities, and gives rise to new sophisticated resource sharing schemes that yield more efficient utilization of radio spectrum for secondary users, regardless of primary user activity.
\end{abstract}

\section{KEYWORDS}

Internet of Things, Cognitive Radio networks, Dynamic Spectrum Access, Behavioral-Ecological networks, self-coexsitence.

\section{INTRODUCTION}

Opportunities afforded by cheaper hardware, more ubiquitous wireless services, and increased demand for end-user applications, to gether have led to rapid population growth in the Internet of things

Permission to make digital or hard copies of all or part of this work for personal or classroom use is granted without fee provided that copies are not made or distributed for profit or commercial advantage and that copies bear this notice and the full citation on the first page. To copy otherwise, to republish, to post on servers or to redistribute to lists, requires prior specific permission and/or a fee. BICT 2017, March 15-16, Hoboken, United States ISBN 978-1-63190-148-5

DOI: 10.4108/eai.22-3-2017.152395

Copyright $\odot 2017$ EAI

\author{
Mohammad Abu Shattal \\ Electrical and Computer Engineering \\ Western Michigan University \\ Kalamazoo, MI 49008, U.S.A \\ mohammad.a.shattal@wmich.edu
}

(IoT). This trend is expected to significantly impact device coexistence due to limited spectrum availability. Although IoT most often is thought of as low power sensors with limited hardware capabilities, the need to support Big Data transmission is inevitable. As wireless devices become more sophisticated, Dynamic Spectrum Access (DSA) networks [15] using Cognitive Radio (CR) technology offers a potential solution to relieve over-crowded wireless channels as has been argued in [40], [45], [66], [14].

Current spectrum assignment policies have resulted in suboptimal use of spectral resources [55]-over-utilization in some bands and under-utilization in others $[3,23,24]$. Few segments of spectrum remain for new services, and yet, the licensed spectrum appears to be underutilized. The reason for this apparent paradox is that licensed ("primary") users are idle within their bands for significant fraction of time [22] leaving behind a "spectrum hole" [31]. This phenomenon has motivated spectrum policy reforms by the U.S. Federal Communications Commission (FCC), including DSA. The objective of DSA-resolving suboptimal spectral resource utilization-is to be realized through recently proposed Cognitive Radio (CR) concepts $[1,39]$. CR nodes dynamically identify and opportunistically forage for unused spectrum bands, adjusting transmission/reception parameters accordingly [1, 7, 15, 16, 25, 29].

The most important constraint on spectrum foraging is that $\mathrm{CR}$ nodes must not interfere with the primary user who is the license holder of the band; that is, upon the primary user's return, CRs must switch to another band. Modulo this constraint, multiple CR networks operated by a multitude of WSPs compete with each other as secondary users (SUs) of limited spectrum, seeking efficiency and quality of service (QoS). Although the FCC reforms "allow unlimited numbers of unlicensed [secondary] users to share frequencies", it "does not provide any right to protection from interference" [23, 24]. Since there are many secondary users, each SU's selection of band and decision to transmit, potentially impacts other secondary users, whose channel bandwidth degrades when greater numbers of SUs share a channel.

Taken together, channel scarcity, usage dynamism, and "no right to protection from interference" present serious challenges to selfcoexistence and performance for CR units in secondary DSA networks $[5,12,26,51]$. Given that CR networks in DSA environments 
are autonomous and (at least in theory) capable of sensing, learning, and adaptation, they may evolve over time, much as humans and other social animal species have in analogous contexts of resource sharing/conflict [9, 27, 28, 61]. Mapping the most plausible evolutionary trajectories of IoT CR societies is subject of inquiry in the present work.

\section{PRIOR WORK}

Given that the FCC's open access paradigm only mandates that secondary users (SUs) do not interfere with primary user (PU) transmission, most prior research has focused on the interaction of PUs with SUs. In particular, spectrum sensing has been applied in this context, to detect primary user arrival and departure (see [65] for a survey of results). By sensing spectrum holes (frequencies where the primary user is presently inactive), secondary users can find bands that are available for opportunistic use [57]. While many researchers have developed solutions for spectrum sensing [31, 64, 65] in the context of PU-SU dynamics, relatively few researchers have looked at the implications and issues surrounding SU-SU interactions (see $[62,67]$ ). Non-cooperative game theory has been used extensively in prior work, to describe the competition between secondary users over a limited amount of resources [42, 52]. Unfortunately (see Xu et. al., and others), a frequent limitation of these approaches is that the game is repeatedly played for just one step [63].

Computer science research on resource allocation in networks recognizes the potential relevance of knowledge on resource use in human and animal societies; for a recent survey of bio-socially inspired approaches, see [21, 38, 41], and books [43, 60]. There has been considerable prior work seeking to apply models of animal foraging strategies (and derivative theories of marginal use) to the design of protocols in the Internet $[36,44,58]$, towards routing and management in mobile ad-hoc networks $[8,11,13,20,34,35,49]$, within sensor networks $[6,10,30,50]$, and now most recently, in the domain of cognitive radio (CR) networks [2, 19, 37, 47, 48]. Previous bio-inspired approaches have depended on inductive analyses of biosocial foraging, wherein idealized formal models are proposed for animal societies (e.g. termites, ants, birds, bacteria, etc.)-a strategy whose weakness was recognized long ago [54]. These inductively derived models of resource consumption were then applied to the design of networking protocols, e.g. termites [49], ants [46], birds [18], bacteria [11] etc. In assuming an inductively derived model of behavior, previous bio-inspired advances assume a basic level of coordination, namely that the individuals in the networked society abide by a parametrized set of rules of resource-sharing that have been agreed upon beforehand. As such, these approaches fail to recognize the long-term evolutionary optimization processes that underlie and give rise to the observed structural patterns of co-use within specific instances of bio-social collectivities-our perspective here precisely addresses this omission.

\section{OUR APPROACH}

In this paper, we evaluate the hypothesis that $\mathrm{CR}$ evolution could plausibly be expected to parallel the evolution of animal species in conditions of resource sharing and conflict $[28,61]$. Towards this, we put forward four successively more sophisticated biologically inspired evolutionary stages of CR capabilities: non-foraging, foraging, contention-sensing, and sociality. The four stages are listed briefly below, and described at length in the next section.

- Evolutionary Stage 1: “Consuming”. SUs are always consuming bandwidth in some spectrum hole, and autonomously decide when to switch channels (e.g. when a PU arrives).

- Evolutionary Stage 2: "Foraging". SUs can either consume a channel's bandwidth or be silent ("forage"). They autonomously decide when to transition between these two states and when to switch channels.

- Evolutionary Stage 3: “Contention-Sensing”. SUs can estimate the number of other SUs that are concurrently consuming the channel. This environmental data biases their decision on when to transition between forage/consume states and when to switch channels.

- Evolutionary Stage 4: "Sociality". SUs can sense some characteristics of other SUs that are concurrently consuming the channel. This social data biases their decision on when to transition between forage/consume states and when to switch channels.

First, we develop a general parametric behavioral model which encompasses the four evolutionary stages above; for each stage, a concrete instance of $\mathrm{CR}$ society can be obtained by specializing this general model.

The resulting $\mathrm{CR}$ societies and their associated strategies are then rendered as parametrized non-cooperative mixed-strategy games, extending the ideas put forth by Tan et al. [56]. In contrast to formal analysis of repeated one step game play [63], our evaluation here is based on simulation experiments that determine the performance profiles of secondary users who continuously apply the prescribed strategies over long time intervals.

Finally, by considering heterogeneous societies consisting of two different types of SUs (a majority with more primitive capabilities, and a minority with more advanced capabilities), we quantify the anticipated evolutionary pressures in SU societies facing resource challenges. This makes it possible to deduce the plausibility of the above 4-stage evolutionary trajectory for distributed uncoordinated CR societies, under the standard assumption that its constituent individuals seek to maximize their utility [4,32].

\section{MATHEMATICAL MODEL}

In our model, SUs are either consuming or foraging, and transition stochastically between these two states. Depending on the evolutionary stage being considered, transition probabilities may be fixed, or biased over time by instantaneous environmental and social factors.

We assume a discrete time stochastic system of $n$ secondary users $\mathcal{S}=\left\{z_{1}, z_{2}, \ldots, z_{n}\right\}$ and $m$ orthogonal spectrum bands $\mathcal{B}=$ $\left\{b_{1}, b_{2}, \ldots, b_{m}\right\}$ in which individuals operate according to a twostate finite state machine (FSM) shown in Figure 1 (following [59]). The FSM consists of two states $Q=\left\{q_{c}, q_{f}\right\}$ and one state variable, the band of interest (BoI) which takes a time varying value $b \in \mathcal{B}$.

State $q_{c}$ represents a "consume" state, during which the SU is transmitting in the BoI; $q_{f}$ represents a "forage" state, during which 


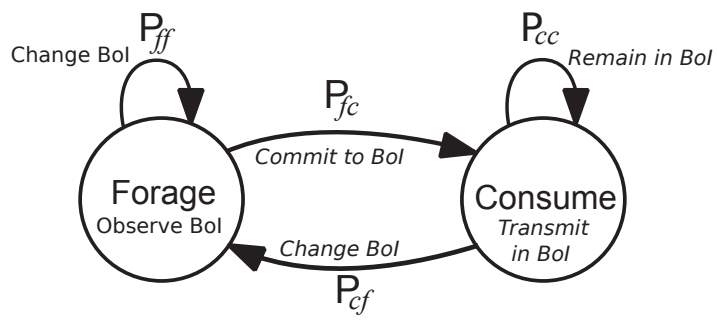

Figure 1: A general behavioral finite state machine for SUs.

the SU is tuned to a BoI but it is not consuming any bandwidth. To keep track of instantaneous state, we introduce a time indexed function

$$
\gamma_{t}: \mathcal{S} \rightarrow\left\{q_{f}, q_{c}\right\}
$$

where $\gamma_{t}(z)$ indicates the state of SU $z$ at time $t$. At time $t$, each $\mathrm{SU} z \in \mathcal{S}$ is either foraging or consuming some band $\rho_{t}(z) \in \mathcal{B}$; this implicitly defines a set of time-indexed maps

$$
\rho_{t}: \mathcal{S} \rightarrow \mathcal{B}
$$

assigning SUs to bands. At time $t$, each $\mathrm{SU} z \in \mathcal{S}$ faces a decision to switch states. If $z$ is consuming at time $t$ (i.e. $\gamma_{t}(z)=q_{c}$ ), then at $t+1$ :

- With probability $P_{c f}$, it switches to a new band $\rho_{t+1}(z)$ chosen uniformly at random, and starts foraging.

- With probability $P_{c c}=1-P_{c f}$, it continues to consume its present band $\rho_{t+1}(z)=\rho_{t}(z)$.

If $z$ is foraging at time $t$ (i.e. $\gamma_{t}(z)=q_{f}$ ), then at $t+1$ :

- With probability $P_{f c}$, it starts consuming its present band $\rho_{t+1}(z)=\rho_{t}(z)$.

- With probability $P_{f f}=1-P_{f c}$, it switches to a new band $\rho_{t+1}(z)$ chosen uniformly at random, and continues foraging.

\subsection{Behavioral Models}

The above mathematical model can be specialized to capture four evolutionary stages of SU capabilities, non-foraging, foraging, contention-sensing, and sociality, discussed in the following subsections.

4.1.1 Evolutionary Stage 1: "Non-foraging". In this stage, SUs are always consuming bandwidth in some spectrum hole, and autonomously decide when to switch channels (e.g. when a PU arrives). This model is widely used in cognitive radio literature (see e.g. [56], [59, 63]).

A stage 1 behavioral model is obtained by tying $P_{f_{c}}=1$ (and hence $P_{f f}=0$ ), yielding the FSM of figure 2 in which the SU will switch directly between bands and, essentially, consume continuously. We refer to this concrete stage 1 society as Non-Foraging (NF).

4.1.2 Evolutionary Stage 2: "Foraging". In this stage, SUs can either consume a channel's bandwidth or be silent ("forage") and autonomously decide when to transition between these two states and when to switch channels. A stage 2 behavioral model is obtained by allowing $P_{f c}$ (and hence $P_{f f}$ ) to be set to any value in

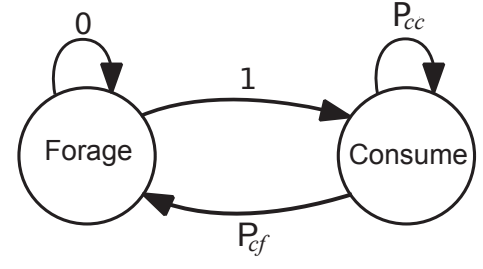

Figure 2: A behavioral finite state machine for SUs in stage 1.

the interval $[0,1]$, so that SU's can inhabit the forage state rather than continuously consume. We refer to this concrete stage 2 behavioral model as Foraging-Blind (FB).

4.1.3 Evolutionary Stage 3: "Contention-Sensing”. In this stage, SUs can estimate the number of other SUs that are concurrently consuming the channel, and this environmental data (which in practice may be obtained at the physical or MAC layers) biases their decision on when to transition between forage/consume states and when to switch channels. Having richer capabilities of this stage, SUs are faced with a range of new spectrum co-use etiquettes, based on the manner in which they react to knowledge of their physical environment.

Foraging with Contention-Sensing is a behavioral model for Stage 3 SUs, obtained by allowing the transition probabilities $P_{f c}, P_{c f} \in[0,1]$ to be biased by contention level in the band of interest:

$$
\mu_{t}(z)=\left|\rho_{t}^{-1}(\rho(z)) \cap \gamma_{t}^{-1}\left(q_{c}\right)\right|
$$

Rather than assuming that an $\mathrm{SU} z$ is able to measure the precise number $\mu_{t}(z)$ of other SUs consuming $\rho(z)$, we only require that it be able to determine whether the band's occupancy is "low" $\mu_{t}(z) \leqslant \tau$ or "high" $\mu_{t}(z)>\tau$ (for the fixed system-wide integer threshold parameter $\tau$ ). If the band has high occupancy, the FSM probabilities are biased by a system-wide parameter $\epsilon \in[0,1]$, making it less likely to be in the consume state and more likely to be in forage state; the reverse bias is manifested when bands have low occupancy (see Figure 3).

We consider three different etiquettes determined by the choice of $\tau$ :

- In the Foraging-Rational (FR) etiquette, each FR consumer is aware of the number of resources $m$ and consumers $n$, taking the rational estimate of the threshold occupancy $\tau=\frac{n}{m}$.

- In the Foraging-Apathetic (FA) etiquette, each FA consumer takes $\tau \gg \frac{n}{m}$ and is thus apathetic to the presence of co-users.

- In the Foraging-Intolerant (FI) etiquette, each FI consumer takes $\tau=0$ and is thus intolerant to contention.

The choice of the variable $\epsilon$ and $\tau$ serve to control how rational, intolerant, or apathetic secondary users are to contention with other SUs at a spectrum hole. If $\epsilon$ is high or $\tau$ is low, the SUs will be more discriminating and thus spend more time scanning for low occupancy bands, and will be more reluctant to leave low occupancy bands. 


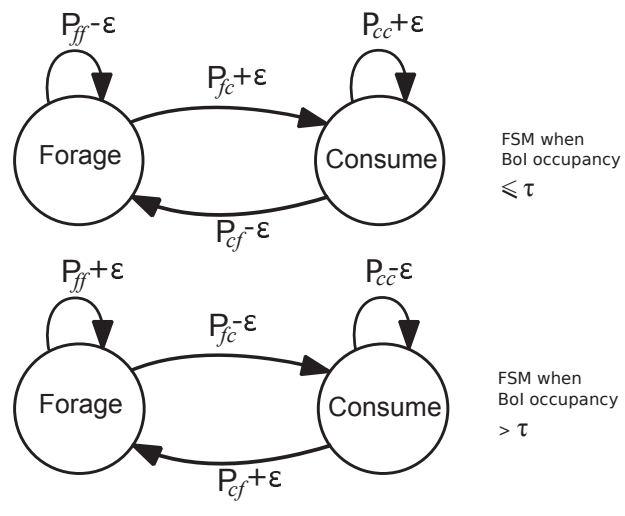

Figure 3: Behavioral FSMs for SUs in stage 3.

4.1.4 Evolutionary Stage 4: "Sociality". In this stage, each SU is additionally capable of sensing some characteristics of the other coconsumers of its current BoI, and this social data biases its decision on when to transition between forage/consume states and when to switch channels. Having richer capabilities at this stage, SUs are faced with a range of new spectrum co-use etiquettes, based on the manner in which they react to knowledge of their social environment.

Towards analyzing the fundamental implications of sociality, we assume that stage 4 SUs have a single differentiating characteristic $\chi$, and an enhanced sensing capability $\mu_{t}^{*}(z)$ by which they estimate the number of the co-consumers that share the same characteristic value. For example, if $\chi$ represented vendor, then each SU would be assumed capable of estimating the number of coconsumers in the band that are from its own vendor. Other candidates for $\chi$ might be organizational affiliation, task, network carrier, etc. - the precise choice is not material to the arguments that follow. Determining such contention levels could be implemented in a distributed manner or through a centralized entity [31, 64, 65, 67] the details of which are beyond the scope of this paper.

In this work, we consider one concrete etiquette governing how an $\mathrm{SU} z$ chooses to react to the knowledge $\mu_{t}^{*}(z)$ of their social environment, splitting based on diversity. In the Diversified (D) behavioral model for Stage 4 SUs, a population $\mathcal{S}$ of $n$ SUs splits into two independent subsystems based on the value of the characteristics $\chi$ (see Figure 4). For simplicity, in this work, we assume that

$$
\chi: \mathcal{S} \rightarrow\{0,1\}
$$

is binary-valued. Each $\mathrm{SU} z$ is assumed to have intrinsic characteristic $\chi(z)$, and can measure the number of co-consumers of its band of interest that share the characteristic value:

$$
\mu_{t}^{*}(z)=\left|\rho_{t}^{-1}\left(\rho_{t}(z)\right) \cap \gamma_{t}^{-1}\left(q_{c}\right) \cap \chi^{-1}(\chi(z))\right| .
$$

If the $n$ SUs are sharing $m$ channels each with bandwidth $B$, the system splits into two independent subsystems: $\mathcal{S}_{0}=\{s \in$ $\mathcal{S} \mid \chi(s)=0\}$ consuming $m$ bands, each of capacity $\frac{\left|\mathcal{S}_{0}\right|}{n} B$, and $\mathcal{S}_{1}=\{s \in \mathcal{S} \mid \chi(s)=1\}$ consuming $m$ bands, each of capacity $\frac{\left|\mathcal{S}_{1}\right|}{n} B$. Figure 4 shows a system of 8 SUs (where 4 have characteristic $\chi=0$ and 4 have $\chi=1$ ) and $m=3$ bands of capacity $B$. The system undergoes a 2-way split, yielding two subsystems of

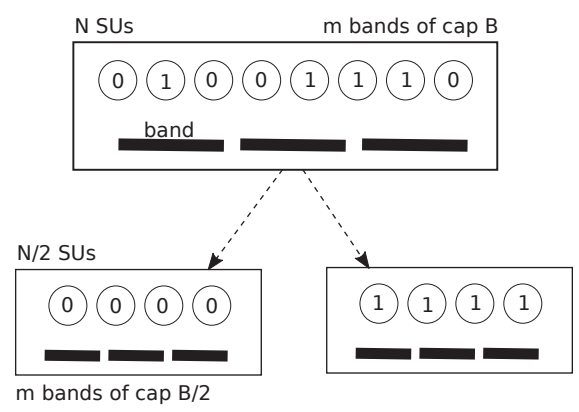

Figure 4: 2-way splitting of consumers/resources.

half the scale (in terms of the number of SUs and channel capacity). The characteristic $\chi$ plays a central role in the differentiation of SUs, making it possible for splitting to occur.

\subsection{Benefit, Costs, and Net Utility}

Since benefit is only obtained when an SU $z$ is in the consume state $q_{c}$, the total system utility is captured by

$$
W_{t}=\sum_{i=1}^{m} k_{t}(i) R\left(k_{t}(i)\right) \text {. }
$$

where

$$
k_{t}(i)=\left|\rho_{t}^{-1}(i) \cap \gamma_{t}^{-1}\left(q_{c}\right)\right|
$$

and

$$
R(k) \stackrel{\text { def }}{=} B \cdot \log _{2}\left(1+\frac{G_{z} P_{z}}{\sum_{y=1}^{k} G_{z y} P_{y}+\omega}\right) .
$$

This definition of benefit (6) is drawn from information theoretic considerations (i.e. Shannon's formula [53]), and is consistent with a significant body of prior work on spectrum sharing [17]. In expression (6), the transmission power for $\mathrm{SU} z$ (resp. $y$ ) are denoted $P_{z}$ (resp. $\left.P_{y}\right) ; B$ is the channel bandwidth; $G_{z}$ represents the channel gain for the transmissions by $z, G_{z y}$ represents the channel gain for the transmission between $z$ and $y$, and $\omega$ is the power level of the ambient white Gaussian noise. To isolate the impact of the proposed paradigms, here we do not consider path losses, and consider a homogeneous network wherein all SUs send (to a base station) at the same power $P$ and experience the channel gain $G$.

When an SU switches transmission bands on entering (or reentering) $q_{c}$, transmitter reconfiguration is required. We assume this to be an expensive operation compared to the receiver reconfigurations required upon entering (or re-entering) $q_{f}$. To capture this, our model charges each SU a fixed cost $c$ whenever it switches transmission bands by moving from the forage state $q_{f}$ to the consume state $q_{c}$. Function $M_{t}$ captures the number of SUs charged for switching at time $t \geqslant 2$

$$
M_{t}=\left\{s \in \mathcal{S} \mid \gamma_{t-1}(s)=q_{f} \wedge \gamma_{t}(s)=q_{c}\right\}
$$

so the switching cost paid by the system at time $t$ is

$$
C_{t}=c\left|M_{t}\right|
$$

and the instantaneous average utility (per SU) at time $t$ is

$$
I_{t}=\frac{1}{n}\left(W_{t}-C_{t}\right)
$$


and the average utility (per SU per unit time) up to time $\mathrm{T}$ is

$$
U_{T}=\frac{1}{T} \sum_{t=1}^{T} I_{t} .
$$

Note that even though the SUs that are in the forage state are excluded from $W$, the utility function implicitly incorporates a zero reward for each SU in the forage state since in (9), the quantity $\left(W_{t}-C_{t}\right)$ is divided by $n$, the total number of SUs in the system.

Remark: We note that given the performance metric incorporates switching cost as a kind of disutility, the utility $U_{T}$ measured in this way is only useful in making relative assessments. That is, one may use $U_{T}$ to make inferences about the relative performance of behavioral scheme A versus B in different regimes, but the absolute numerical values of $U_{T}$ are not directly interpretable in isolation.

\subsection{Emergence and Evolutionary Pressures}

To understand evolutionary pressures on the emergence of new SU capabilities and etiquettes, we evaluate a range of heterogeneous CR societies in which each SU follows one of two distinct behavioral models. The heterogeneous society $(X, Y)_{\alpha}$ one in which $\alpha n$ SUs operate according to model $Y$, while $(1-\alpha) n$ operate according to model $X^{1}$, where $X, Y$ are one of e.g. NF, FB, FR, FI, FA., etc. and $0 \leqslant \alpha \leqslant 1$. By varying $\alpha$ from 0 to 1 , we can evaluate the incentives for or against the emergence of a subsociety following behavioral model $Y$ within an ambient society following behavioral model $X$.

\section{EXPERIMENTAL RESULTS}

We use a stochastic discrete event simulator [33] to obtain utility measurements for different SU societies over time. Typically, in the graphs below, each plotted data point is an experiment, which is repeated for 10 independent trials. Error bars indicate the standard deviation of the measured value. Consumers transition asynchronously according to the appropriate previously defined finite state machines (FSM). To facilitate comparison with the work of Tan and others $[56,59]$ in many experiments, we use the same parameter values as these previous researchers. These values are listed in Table 1 below:

Table 1: Baseline Parameters

\begin{tabular}{|c|l|c|}
\hline Parameter & Description & Value \\
\hline \hline$n$ & Number of SUs & 30 \\
$m$ & Number of bands & 5 \\
$P_{z}$ & Transmission power of node z & $4 \mathrm{~W}$ \\
$B$ & Capacity per band & $20 \mathrm{MHz}$ \\
$C$ & Switching cost & $0.3 \cdot B$ \\
\hline \hline$P_{f c}$ & Prob. of commencing consume & 0.12 \\
$P_{c f}$ & Prob. of commencing foraging & 0.21 \\
$\epsilon$ & Bias based on occupancy & $5 \%$ \\
$\tau$ & Rational occupancy threshold & $n / m$ \\
\hline
\end{tabular}

\footnotetext{
${ }^{1}$ Note that $(X, Y)_{\alpha}$ is equivalent to $(Y, X)_{1-\alpha}$, we limit this ambiguity by adopting the ordering convention that $Y$ is at least as advanced as $X$ (in terms of evolutionary stages and corresponding SU capabilities).
}

\subsection{The Emergence of (Blind) Foraging}

To begin, we describe the circumstances under which foraging capabilities might plausibly emerge within societies of non-foragers and show that this etiquette shift is related to population size. Figure 5 shows the utility achieved in a $(N F, F B)_{0.1}$ society where $10 \%$ of the population is foraging and $90 \%$ is not. We see that when the population grows (but resources are held constant at the baseline values listed in Table 1) there is a critical size at which a blind foraging strategy (FB) outperforms non-foraging behavior (NF). In the specific experiment we conducted, this breakpoint occurred at $n=220$.

When $n \ll 220$, the adoption of foraging behavior is penalized, and hence is expected to be selected against in evolutionary terms. This phenomenon is seen in Figure 6, which considers a population of $n=30$ SUs, of which $\lfloor 30 \cdot \alpha\rfloor$ are blind foragers (while the rest are non-foragers); all other experiment parameters are set as specified in the baseline (see Table 1). We see that for low values of $\alpha$ (e.g. when the first forager emerges) the utility attained by the foraging minority subpopulation (2.9) is significantly smaller than that which is enjoyed by non-foraging majority subpopulation (6.5). The dotted curves in Figure 6 represent a system in which the primary user decides to return to the band $30 \%$ of the time. We can see that even with primary user interruption, non-foragers continue to outperform as the foraging population emerges.

In contrast, when $n \gg 220$, the adoption of foraging behavior is systemically rewarded, and hence is expected to be selected in evolutionary terms. This phenomenon is seen in Figure 7, which considers a population of $n=300$ SUs, of which $\lfloor\alpha \cdot 300\rfloor$ are blind foragers (and the rest are non-foragers). We see that for low values of $\alpha$, in particular, at the moment when the first foragers emerge, the utility attained by the foraging minority subpopulation is greater $(-0.13)$ than that enjoyed by non-foragers $(-0.31)^{2}$. As long as $\alpha<0.55$, foraging outperforms non-foraging. When $n=400$ this critical $\alpha$ value rises from 0.55 to 0.85 . Thus, the longer a growing society waits to move from non-foraging to foraging behavior, the more a widespread change of behavior promises to be in the rational self-interest of individuals therein.

Regardless of $n$, monotonic positive first derivatives with respect to $\alpha$ (Figures 6 and 7) imply a differential increase in utility for both non-foragers and foragers. From this we may conclude that once foraging behavior arises, each individual (regardless of their behavior model) experiences an increase in utility if a greater percentage of the population adopts the foraging model. This positive differential (in populations greater than a critical threshold size), is evidence of the evolutionary pressure towards the emergence and widespread adoption of foraging behavior in CR societies of non-foragers.

\subsection{The Emergence of Sensing and Rationality}

We describe the circumstances in which contention-sensing capabilities (i.e. the FR behavioral model) might plausibly emerge within societies of blind foragers and show that foraging-rational etiquette is advantageous to the first deviating SU in a population

\footnotetext{
${ }^{2}$ See remark at the end of section 4.2 concerning interpretations of utility.
} 


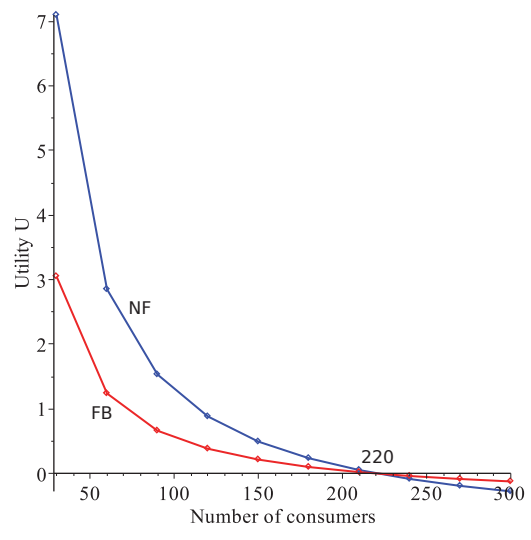

Figure 5: The merits of foraging in growing populations.

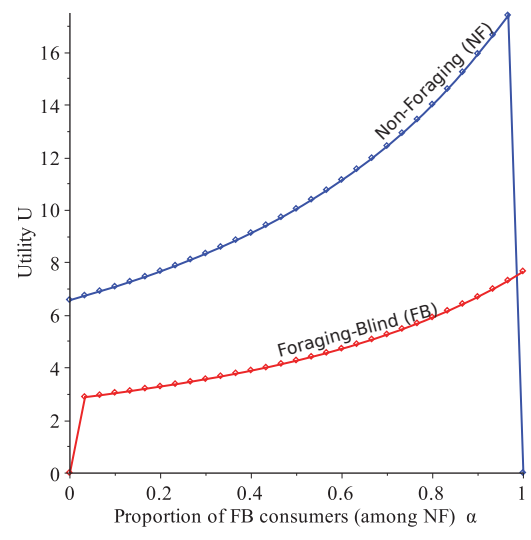

Figure 6: The rise of foraging among nonforagers $(n=30)$.

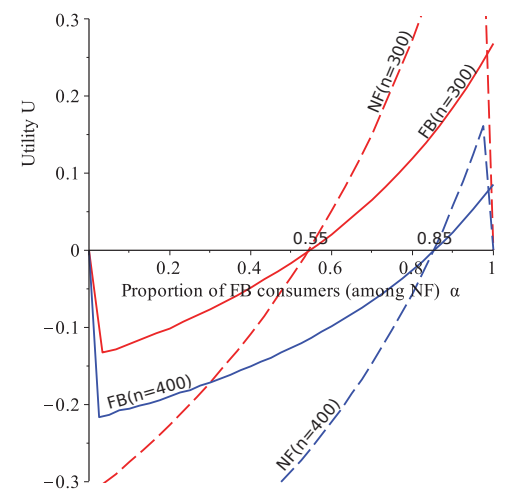

Figure 7: The rise of foraging among nonforagers $(n=300)$ size of 30 and continuous to be advantageous as the size of followers grows. In Figure 8, we consider a population of $n=30$ SUs, of which $\lfloor 30 \cdot \beta\rfloor$ are sensing their environment and responding according to the rational etiquette (FR), while the rest are blind foragers (FB). We see that for low values of $\beta$ (e.g. when the first rational forager appears) the utility attained by FR nodes is significantly greater (11.0) than the mean utility attained by blind foragers (7.6). This systemic reward is experimental evidence of evolutionary pressure towards the emergence of sensing capabilities and rational etiquettes in CR societies of blind foragers. As $\beta$ increases, each SU that changes behavior from blind foraging to rational foraging experiences a benefit (albeit at the expense of everyone else, as implied by the monotonic negative first derivatives with respect to $\beta$ ). The observation continues to hold in a system where the primary user decides to return to the band $30 \%$ of the time (dotted line in Figure 8).

As the population size $n$ increases, foraging-rational continues to outperform foraging-blind in terms of utility and is expected to be selected in evolutionary terms. Figure 9 shows the utility achieved in a $(F B, F R)_{0.1}$ society where $10 \%$ of the population is sensing and following a rational etiquette while $90 \%$ is foraging blindly. We see that the subpopulation that follows a rational etiquette consistently outperforms the larger ambient population of blind foragers at all population sizes. We also see that as the population grows (but resources are held constant) the relative advantage of the FR model increases. For example, when $n=30$ the advantage was $145 \%$ (11.0/7.6); by the time the population grows to $n=300$ the minority's advantage from using the FR model (compared to the ambient population using the FB model), is $250 \%$ $(0.5 / 0.2)$. This indicates that the evolutionary pressure to adoption a rational sensing model increases as the total population grows larger. The above analysis demonstrates that at some scale, there will be evolutionary pressure to transition from foraging blindly to foraging in a rational manner based on band occupancy sensing.

5.2.1 Dynamic Estimation of Population Size. In FR societies, the etiquette of "rationality" mandates that each SU set its occupancy threshold to $\tau=n / m$, and thus requires each SU to know (through offline knowledge) both the size of the population $n$ and the number of resources $m$. This can be a problematic assumption in growing SU societies. Here we consider a dynamic estimation scheme for $n$ based on random sampling of channel occupancies.

Estimation scheme. Each SU dynamically estimates the total number of consumers $\tilde{n}$ over a time window $\Delta$ by taking:

$$
\tilde{n}_{t}=m \cdot \frac{1}{\Delta} \sum_{\delta=0}^{\Delta-1} \mu_{t-\delta(z)}
$$

and then setting its threshold occupancy level to $\tau_{t}=\frac{\tilde{n}_{t}}{m}$. Figure 10 shows that such dynamic estimation of population sizes is successful in quickly converging to the performance of systems that are pre-configured with offline knowledge about the true population size $n$. The dynamic FR scheme has the advantage that it requires less startup configuration and can be used in situations where the population size is either unknown or time varying.

\subsection{Alternative Etiquettes in Societies with Sensing}

In what follows, we consider the evolutionary pressure for or against the emergence of sub-populations that deviate from the rational etiquette, within an FR society. We show that foraging-intolerant (FI) and foraging-apathetic (FA) strategies are not advantageous in a foraging-rational (FR) society and will be selected against in evolutionary terms. Recall that "rationality" of FR behavior is defined in terms of SUs taking the threshold occupancy $\tau=n / m$ (where $n$ may be dynamically estimated as described in the previous section). Here we consider extreme deviations from this value. For simplicity, we consider two cases: first, the "Apathetic" etiquette, in which the threshold is taken to be much higher than rational $\tau=2 n / m$; second, we consider the emergence of "Intolerant" etiquette, in which $\tau=0$. These two alternative etiquettes are treated in subsections (a) and (b) below, respectively. As we shall see, based on evolutionary considerations, neither is expected to emerge. 


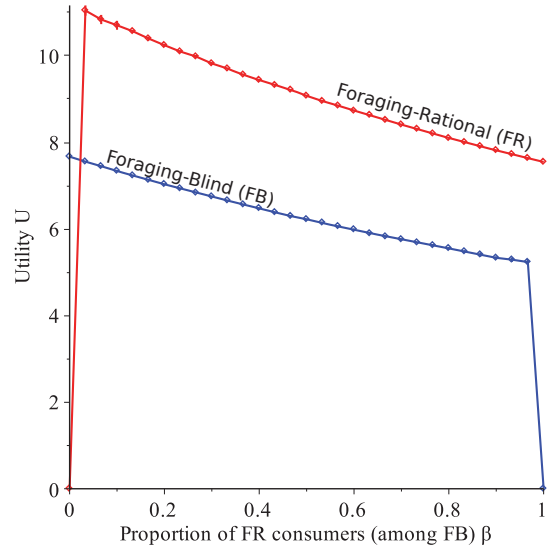

Figure 8: The emergence of sensing and rationality.

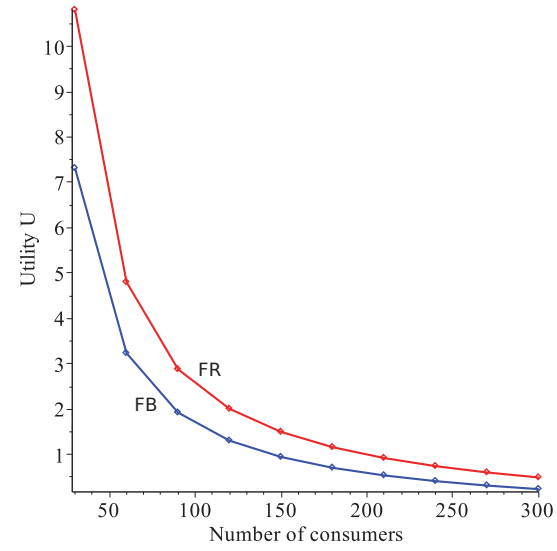

Figure 9: The merits of rationality in growing populations.

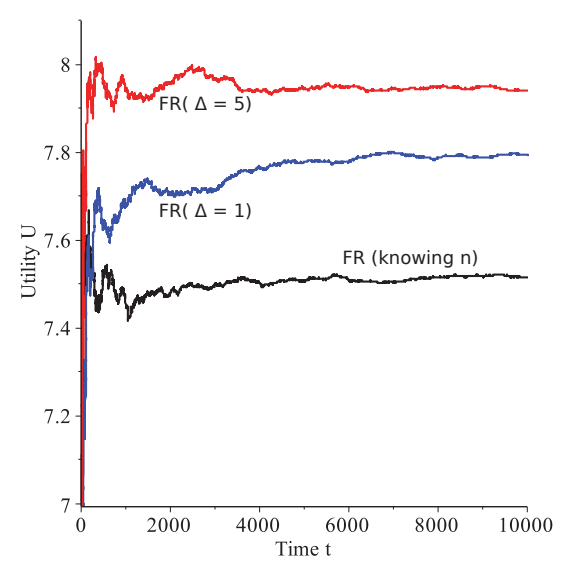

Figure 10: FR with dynamic estimate of population size.
The Etiquette of Apathy. Figure 11 shows the change in utility for rational and apathetic societies as the proportion of apathetic consumers increases. As can be seen, regardless of their proportion within the population, apathetic SUs do not gain any advantage over rational consumers (7.5) by choosing a higher than rational threshold.

The Etiquette of Intolerance. In the intolerant etiquette, an SU chooses to set their occupancy threshold $\tau$ to be lower than rational. Here we consider the extreme case, when $\tau=0$.

As the population grows to a certain size, foraging-intolerant continues to be selected agains in evolutionary terms. Figure 13 shows the utility achieved in a $(F R, F I)_{0.1}$ society where $10 \%$ of the population is following an intolerant etiquette while $90 \%$ is foraging rationally. We see that a rational etiquette consistently outperforms the minority of intolerant foragers at all population sizes. We also see that as the population grows (but resources are held constant) the relative advantage of the FR model over the FI model, increases. For example, when $n=30$ the advantage was $210 \%$ (7.6/3.4); by the time the population grows to $n=300$ the minority's advantage from using the FR model (compared to the ambient population using the FI model), is $4500 \%(0.45 / 0.01)$. This indicates that the evolutionary pressure against an intolerance etiquette increases as the total population grows larger. The above analysis demonstrates beyond some scale, there will be significant evolutionary pressure against deviations from rational etiquette. We note that in the baseline experiment (see Figure 12), the utility for FI does eventually exceed FR but only after a very substantial fraction of the population has switched to FI behavior, $\eta>0.9$. This threshold exhibits a significant evolutionary barrier against transitions from FR to FI. We note the same behavior in a system where the primary user decides to return to the band $30 \%$ of the time (see dotted curves in Figure 12).

The structural explanation for why the transition to FI is impeded can be best understood by examining Figure 14. Here we consider a minor perturbation of the baseline experiment settings (see Table 1). Specifically, we consider a society of 29 standard
FR nodes (who set rational threshold $\tau=n / m=30 / 5=6$ ), together with one non-standard deviant player who chooses to set their threshold $\tau$ autonomously. The graph shows what the utility of the deviant player is (compared to the utility of the law-abiding standard FR majority), for each possible nonstandard setting of $\tau$ that might be used. Utilities obtained when $\tau=0$ (intolerant) and $\tau=12$ (apathetic) are consistent with figure 12 and figure 11 for low values of $\eta$ and $\xi$ respectively. What we see is that any unilateral deviation from the rationally determined value may result in a performance penalty and never yields an advantage.

\subsection{The Emergence of Diversity for Subdivision}

In this section, we consider the potential benefits of SU diversity, assuming that $\mathrm{CR}$ nodes each have an intrinsic characteristic $\chi$, which can be sensed, and used to implement splitting. We show that emergence of SU diversity in the splitting context is only beneficial for specific population sizes. There are of course countless other ways in which diversity could be harnessed socially-here we consider whether splitting, by itself, presents enough advantages to predict the plausible emergence of SU diversity. In effect, we view splitting as the analogue of ecological niche formation in the $\mathrm{CR}$ domain. The question we are asking then is: Does niche formation produce advantages which would justify the emergence of species diversity $\chi$ among CRs?

How is the benefit obtained by splitting impacted by the system's size? Figure 15 shows the performance advantage of splitting a society of $n$ nodes into two societies of $n / 2$ nodes each receiving $m$ bands of capacity $B / 2$ (normalized by the performance attained in societies which do not choose to split). For example, when $m=10$ and $B=20$ there is a critical value of system size $n=80$ beyond which splitting yields a marginal advantage - but this advantage which is maximal (9\% at $n=120)$ vanishes for $n \gg 80$. The same qualitative behavior is seen for other experiment settings ( $m=15$ and $m=5$ ), albeit with different local maxima and rates of asymptotic convergence to 1 . We conclude 


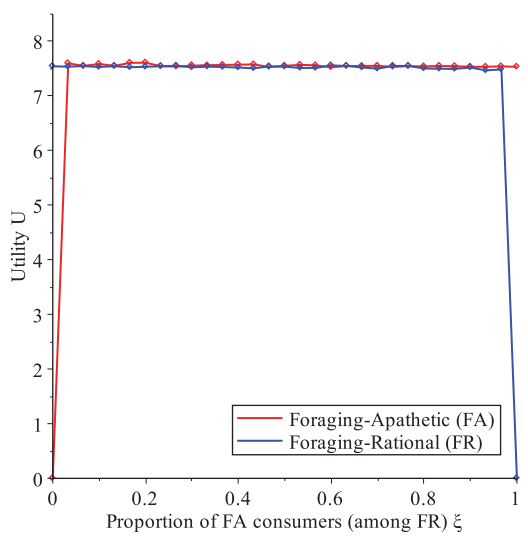

Figure 11: The futility of apathy.

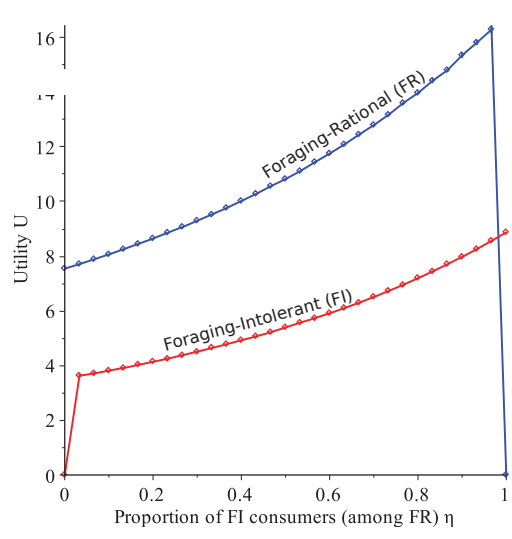

Figure 12: The disincentives to intolerance

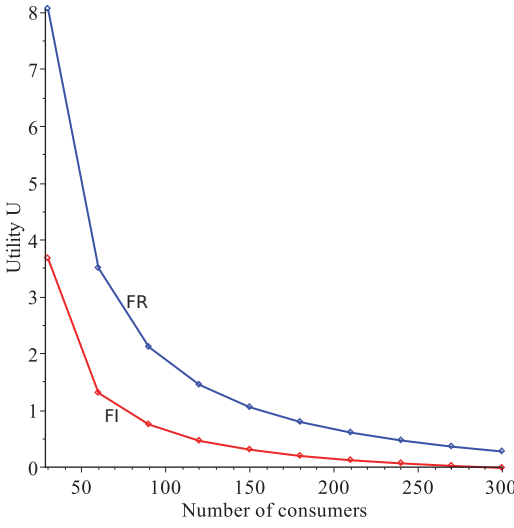

Figure 13: Disincentives to intolerance in growing populations.

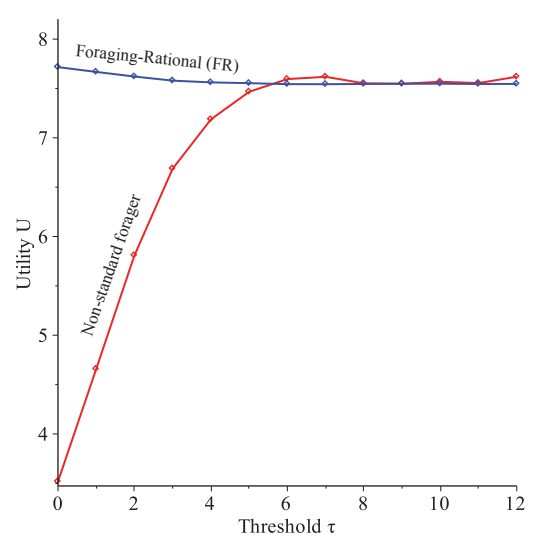

Figure 14: A non-standard forager in an FR society.

that splitting only makes sense in a critical range of society sizeif a society is too small, or too large, no benefit is derived from splitting.

\section{CONCLUSIONS}

In this paper, we considered an evolutionary sequence of increasingly sophisticated capabilities for CR nodes in societies of secondary users of radio spectrum as the IoT population adjusts to saturated resources.

We showed that:

- In a society of SUs which are constantly consuming (stage 1), when the capability to forage for spectrum emerges, there is an evolutionary pressure for a subpopulation to switch to a foraging etiquette, and this subpopulation is expected to grow. This was shown in Section 5.1.

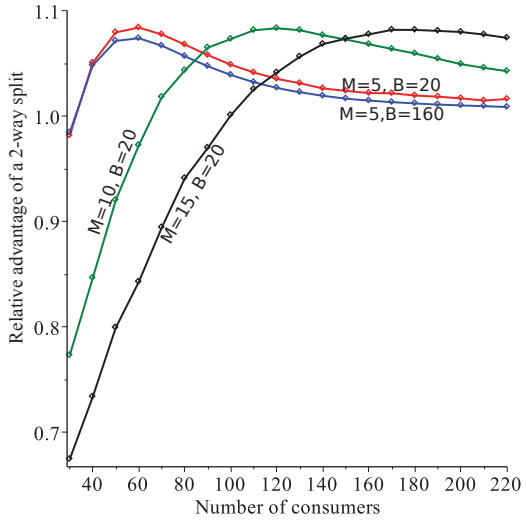

Figure 15: Benefit of a single 2-way split versus society size.

We saw that at a critical population size it becomes advantageous (with respect to utility) for an SU to unilaterally adopt the more advanced strategy. This "first conversion" acts as a seed, since any increase in the fraction of SUs following the new strategy yields a differential improvement in the utility of all SUs (regardless of strategy) thus driving the "upgrading" of the entire population. Next, we saw that:

- In a society of foraging SUs (stage 2), when the capability to sense channel characteristics emerges, there is an evolutionary pressure for a subpopulation to bias transition probabilities accordingly to rational expectations of contention levels. This was shown in Section 5.2.

Regardless of population size, we saw that it becomes advantageous (with respect to utility) for an SU to unilaterally adopt the more advanced strategy.

- In a population of contention sensing foragers, it is not advantageous for a subpopulation to deviate from the rational contention threshold by adopting an attitude of apathy or intolerance to contention. This was seen in Section 5.3. 
Additionally, in Section 5.2.1 we demonstrated that the rational threshold can be estimated dynamically by the SUs, making FR viable in growing populations.

Although many etiquettes based on sociality are conceivable, here we considered one based on splitting.

- Division based on a boolean differentiating characteristic is marginally profitable up to a certain population sizes; larger diverse societies do not profit by splitting into homogeneous subsocieties. This was seen in Section 5.4.

In practice of course, the evolutionary selection of device capabilities and behavioral strategies arises from consumer pressure on manufacturers to deliver increased bandwidth for a rapidly growing IoT device population. Taken together, the projections presented in this paper determine a quantifiably plausible evolutionary trajectory for IoT devices adopting cognitive radio capabilities (and corresponding behavioral strategies) that one might expect to see implemented by manufacturers as they compete for increasing numbers of consumers with increasing bandwidth requirements.

\subsection{Future Work}

We seek to ascertain the extent to which the phenomena reported in this paper are replicable in real hardware testbed of softwaredefined radio systems. Our plan is to experiment with the different evolutionary stages using ns-3 in conjunction with different channel models to provide more insight into the practical implications of the evolutionary stages. In addition, we plan to experiment with actual IoT nodes that utilize a low power microcontroller with the capability to switch the Wi-Fi channel to study the evolution behavior experimentally.

The general question on whether the emergence of CR diversity is evolutionarily selected for remains open. Answering it requires deeper exploration of social structures by which $\mathrm{CR}$ societies might harness node diversity in a manner that benefits its members. The study of more complex social structures supported by differentiation and sensing is an area of active research.

\subsection{Acknowledgements}

This project was supported by a grant from the National Science Foundation program for Enhancing Access to Radio Spectrum (\#1443985), supported by the Directorates for Mathematical and Physical Sciences (MPS), Engineering (ENG), and Computer and Information Science and Engineering (CISE). The opinions, findings, and conclusions or recommendations expressed in this publication are those of the authors and do not necessarily reflect those of the National Science Foundation. The authors would like to thank Dr. Spiros Bakiras, Dr. Shweta Jain, and Dr. Shamik Sengupta for their feedback and discussion relating to the ideas underlying this research, at various stages during its development.

\section{REFERENCES}

[1] I. Akyildiz, W. Lee, M. Vuran, and S. Mohanty. Next generation/dynamic spectrum access/cognitive radio wireless networks: a survey. Computer Networks, 50(13):2127-2159, 2006

[2] B. Atakan and O. B. Akan. Biologically-inspired spectrum sharing in cognitive radio networks. In Wireless Communications and Networking Conference, 2007. WCNC 2007. IEEE, pages 43-48, 2007.
[3] C. Bazelon. Licensed or unlicensed: The economic considerations in incremental spectrum allocations. New Frontiers in Dynamic Spectrum Access Networks, 2008. DYSPAN 2008. 3rd IEEE Symposium on, pages 1-8, Oct. 2008.

[4] G. Belovsky. Insights for caribou/reindeer management using optimal foraging theory. Rangifer, 11(4):7-23, 2010.

[5] K. Bian and J.-M. Park. A coexistence-aware spectrum sharing protocol for 802.22 wrans. pages $1-6$, aug. 2009.

[6] M. Britton, V. Shum, L. Sacks, and H. Haddadi. A biologically-inspired approach to designing wireless sensor networks. In Proceeedings of the Second European Workshop on Wireless Sensor Networks, 2005, pages 256-266, 2005.

[7] S. Buljore, H. Harada, S. Filin, P. Houze, K. Tsagkaris, O. Holland, K. Nolte, T. Farnham, and V. Ivanov. Architecture and enablers for optimized radio resource usage in heterogeneous wireless access networks: The ieee 1900.4 working group. Communications Magazine, IEEE, 47(1):122-129, january 2009.

[8] O. Castillo, R. Martínez-Marroquín, P. Melin, F. Valdez, and J. Soria. Comparative study of bio-inspired algorithms applied to the optimization of type- 1 and type-2 fuzzy controllers for an autonomous mobile robot. Information Sciences, 192:19-38, June 2012

[9] K. Challapali, C. Cordeiro, and D. Birru. Evolution of spectrum-agile cognitive radios: first wireless internet standard and beyond. 2006.

[10] C. Charalambous and S. Cui. A biologically inspired networking model for wireless sensor networks. IEEE Network, 24(3):6-13, 2010

[11] J. Chen and A. Sayed. Bio-inspired cooperative optimization with application to bacteria motility. In 2011 IEEE International Conference on Acoustics, Speech and Signal Processing (ICASSP), pages 5788-5791, 2011.

[12] T. Chen, H. Zhang, M. Hoyhtya, and M. Katz. Spectrum self-coexistence in cognitive wireless access networks. pages 1 -6, 30 2009-dec. 42009.

[13] F. Chiang, J. Agbinya, T. A. T. N. Zealand), and A. C. (New. Bio-inspired topology convergence algorithms in resource-constrained VANETs. 2007.

[14] T. Chiwewe, C. Mbuya, and G. Hancke. Using cognitive radio for interferenceresistant industrial wireless sensor networks: An overview. 11(6):1466-1481.

[15] C. Cordeiro, K. Challapali, D. Birru, and S. Shankar. IEEE 802.22: The first worldwide wireless standard based on cognitive radios. Proc., IEEE Symposium of New Frontiers in Dynamic Spectrum Access Networks (DySPAN'2005), pages 328-337, Nov. 2005.

[16] C. Cordeiro, K. Challapali, D. Birru, and S. Shankar. IEEE 802.22: the first worldwide wireless standard based on cognitive radios. IEEE International Symposium on Dynamic Spectrum Access Networks, pages 328-337, Nov. 2005.

[17] T. M. Cover and J. A. Thomas. Elements of Information Theory. John Wiley \& Sons, July 2006.

[18] P. Di Lorenzo and S. Barbarossa. Distributed resource allocation in cognitive radio systems based on social foraging swarms. In 2010 IEEE Eleventh International Workshop on Signal Processing Advances in Wireless Communications (SPAWC), pages $1-5,2010$.

[19] P. Di Lorenzo, S. Barbarossa, and A. Sayed. Bio-inspired decentralized radio access based on swarming mechanisms over adaptive networks. IEEE Transactions on Signal Processing, Early Access Online, 2013

[20] F. Dressler and O. Akan. Bio-inspired networking: from theory to practice. IEEE Communications Magazine, 48(11):176-183, 2010.

[21] F. Dressler and O. B. Akan. A survey on bio-inspired networking. Computer Networks, 54(6):881-900, Apr. 2010

[22] A. Durantini and M. Martino. The spectrum policy reform paving the way to cognitive radio enabled spectrum sharing. Telecommunications Policy, 37(2-3):8795, Mar. 2013.

[23] FCC. Spectrum policy task force report. pages 02-155, Nov 2002

[24] FCC. In the matter of unlicensed operation in the TV broadcast bands. Second Report and Order and Memorandum Opinion and Order, (FCC-08-260A1), Nov. 2008.

[25] G. Ganesan and Y. Li. Cooperative spectrum sensing in cognitive radio networks. in Proc. of IEEE DySPAN, pages $137-143,2005$

[26] V. Gardellin, S. Das, and L. Lenzini. A fully distributed game theoretic approach to guarantee self-coexistence among wrans. pages $1-6$, march 2010

[27] F. Ge, Q. Chen, Y. Wang, C. Bostian, T. Rondeau, and B. Le. Cognitive radio: From spectrum sharing to adaptive learning and reconfiguration. pages $1-10$, march 2008.

[28] S. Haykin. Cognitive radio: brain-empowered wireless communications. Selected Areas in Communications, IEEE fournal on, 23(2):201 - 220, feb. 2005.

[29] K. Hong, S. Sengupta, and R. Chandramouli. Spiderradio: An incumbent sensing implementation for cognitive radio networking using ieee 802.11 devices. IEEE International Conference on Communications (ICC), pages 1-5, may. 2010.

[30] S. Iyengar, H.-C. Wu, N. Balakrishnan, and S.-Y. Chang. Biologically inspired cooperative routing for wireless mobile sensor networks. IEEE Systems fournal, 1(1):29-37, 2007

[31] C. Jiang, Y. Chen, Y. Gao, and K. J. R. Liu. Joint spectrum sensing and access evolutionary game in cognitive radio networks. IEEE Transactions on Wireless Communications, 12(5):2470-2483, May 2013.

[32] H. Kaplan, P. Hooper, and M. Gurven. The evolutionary and ecological roots of human social organization. Philosophical Transactions of the Royal Society B: 
Biological Sciences, 364(1533):3289-3299, 2009.

[33] B. Khan, K. Dombrowski, and M. Saad. A stochastic agent-based model of pathogen propagation in dynamic multi-relational social networks. SIMULATION,Transactions of SCS, 90(4):460-484, 2014.

[34] Z. Liu, M. Kwiatkowska, and C. Constantinou. A biologically inspired congestion control routing algorithm for MANETs. In Third IEEE International Conference on Pervasive Computing and Communications Workshops, 2005. PerCom 2005 Workshops, pages 226-231, 2005.

[35] Z. Liu, M. Kwiatkowska, and C. Constantinou. A biologically inspired QoS routing algorithm for mobile ad hoc networks. International fournal of Wireless and Mobile Computing, 4(2):64-75, Jan. 2010

[36] Q. Mahmoud. Cognitive Networks: Towards Self-Aware Networks. John Wiley \& Sons, Aug. 2007

[37] X. Mao and H. Ji. Biologically-inspired distributed spectrum access for cognitive radio network. In 2010 6th International Conference on Wireless Communications Networking and Mobile Computing (WiCOM), pages 1-4, 2010.

[38] M. Meisel, V. Pappas, and L. Zhang. A taxonomy of biologically inspired research in computer networking. Computer Networks, 54(6):901-916, Apr. 2010.

[39] J. Mitola and G. Maguire. Cognitive radio: Making software radios more personal. IEEE Personal Commun., 6(4):13-18, Aug. 1999.

[40] M. Murroni, R. Prasad, P. Marques, B. Bochow, D. Noguet, C. Sun, K. Moessner, and H. Harada. IEEE 1900.6: spectrum sensing interfaces and data structures for dynamic spectrum access and other advanced radio communication systems standard: technical aspects and future outlook. 49(12):118-127.

[41] T. Nakano. Biologically inspired network systems: A review and future prospects. IEEE Transactions on Systems, Man, and Cybernetics, Part C: Applications and Reviews, 41(5):630-643, 2011

[42] D. Niyato and E. Hossain. Competitive pricing for spectrum sharing in cognitive radio networks: Dynamic game, inefficiency of nash equilibrium, and collusion. IEEE Journal on Selected Areas in Communications, 26(1):192-202, Jan. 2008.

[43] S. Olariu and A. Y. Zomaya, editors. Handbook of Bioinspired Algorithms and Applications. Chapman and Hall/CRC, 1 edition, Sept. 2005

[44] K. Park and W. Willinger. The Internet As a Large-Scale Complex System. Oxford University Press, 2005

[45] H. Pohls, V. Angelakis, S. Suppan, K. Fischer, G. Oikonomou, E. Tragos, R. Diaz Rodriguez, and T. Mouroutis. RERUM: Building a reliable IoT upon privacy- and security- enabled smart objects. In 2014 IEEE Wireless Communications and Networking Conference Workshops (WCNCW), pages 122-127.

[46] C. R. Reid, D. J. T. Sumpter, and M. Beekman. Optimisation in a natural system: Argentine ants solve the towers of hanoi. The fournal of Experimental Biology, 214(1):50-58, Jan. 2011. PMID: 21147968.

[47] T. Renk, C. Kloeck, D. Burgkhardt, F. K. Jondral, D. Grandblaise, S. Gault, and J.C. Dunat. Bio-inspired algorithms for dynamic resource allocation in cognitive wireless networks. Mobile Networks and Applications, 13(5):431-441, Oct. 2008.

[48] C. J. Rieser. Biologically inspired cognitive radio engine model utilizing distributed genetic algorithms for secure and robust wireless communications and networking. http://scholar.lib.vt.edu/theses/available/etd-10142004023653/, Oct. 2004

[49] M. Roth and S. Wicker. Termite: ad-hoc networking with stigmergy. In IEEE Global Telecommunications Conference, 2003. GLOBECOM '03, volume 5, pages 2937-2941 vol.5, 2003

[50] S. Selvakennedy, S. Sinnappan, and Y. Shang. A biologically-inspired clustering protocol for wireless sensor networks. Computer Communications, 30(1415):2786-2801, Oct. 2007

[51] S. Sengupta, R. Chandramouli, S. Brahma, and M. Chatterjee. A game theoretic framework for distributed self-coexistence among IEEE 802.22 networks. IEEE Global Telecommunications Conference (GLOBECOM), pages 1-6, nov. 2008.

[52] S. Sengupta, R. Chandramouli, S. Brahma, and M. Chatterjee. A game theoretic framework for distributed self-coexistence among IEEE 802.22 networks. In Global Telecommunications Conference, 2008. IEEE GLOBECOM 2008. IEEE, pages $1-6,2008$.

[53] C. E. Shannon. Communication in the presence of noise. Proc. Institute of Radio Engineers, 37(1):10-21, 1949

[54] E. A. Smith. Anthropological applications of optimal foraging theory: a critical review. Current Anthropology, 24(5):625-651, 1983.

[55] Y. Tan, S. Sengupta, and K. P. Subbalakshmi. Competitive spectrum trading in dynamic spectrum access markets: A price war. In Global Telecommunications Conference (GLOBECOM 2010), 2010 IEEE, pages 1-5, 2010.

[56] Y. Tan, S. Sengupta, and K. P. Subbalakshmi. Human society inspired dynamic spectrum access networks: The effect of parochialism. In Global Telecommunications Conference (GLOBECOM 2011), 2011 IEEE, pages 1-5, 2011.

[57] B. Wang and K. J. R. Liu. Advances in cognitive radio networks: A survey. IEEE fournal of Selected Topics in Signal Processing, 5(1):5-23, Feb. 2011.

[58] M. Wang and T. Suda. The bio-networking architecture: a biologically inspired approach to the design of scalable, adaptive, and survivable/available network applications. In 2001 Symposium on Applications and the Internet, 2001. Proceedings, pages 43-53, 2001.
[59] A. Wisniewska and B. Khan. Contention-sensing and dynamic spectrum co-use in secondary user cognitive radio societies. In Wireless Communications and Mobile Computing Conference (IWCMC), 2014 International, pages 157-162, Aug. 2014.

[60] Y. Xiao. Bio-Inspired Computing and Networking. CRC Press, 1 edition, Mar. 2011.

[61] Y. Xing and R. Chandramouli. Human behavior inspired cognitive radio network design. Communications Magazine, IEEE, 46(12):122 -127, december 2008.

[62] Y. Xing and R. Chandramouli. Human behavior inspired cognitive radio network design. IEEE Communications Magazine, 46(12):122-127, Dec. 2008.

[63] Y. Xu, A. Anpalagan, O. Wu, L. Shen, Z. Gao, and J. Wang. Decision-theoretic distributed channel selection for opportunistic spectrum access: Strategies, challenges and solutions. IEEE Communications Surveys \& Tutorials, pages 1-25, 2013.

[64] F. R. Yu, M. Huang, and H. Tang. Biologically inspired consensus-based spectrum sensing in mobile ad hoc networks with cognitive radios. Network, IEEE, 24(3):26-30, 2010.

[65] Y. Zeng, Y.-C. Liang, A. T. Hoang, and R. Zhang. A review on spectrum sensing for cognitive radio: Challenges and solutions. EURASIP fournal on Advances in Signal Processing, 2010:1-16, 2010.

[66] P. Zhang, Y. Liu, Z. Feng, Q. Zhang, Q. Li, and D. Xu. Intelligent and efficient development of wireless networks: A review of cognitive radio networks. 57(28):3662-3676.

[67] J. Zhao and X. Wang. Channel sensing order in multi-user cognitive radio networks. In Dynamic Spectrum Access Networks (DYSPAN), 2012 IEEE International Symposium on, pages 397-407, 2012. 